SNUTP 00-010

\title{
Exact Cosmological Solution and Modulus Stabilization in the Randall-Sundrum Model with Bulk Matter
}

\author{
Jihn E. Kim * and Bumseok Kyae ${ }^{\dagger}$ \\ Department of Physics and Center for Theoretical Physics, Seoul National University, Seoul \\ 151-742, Korea
}

\begin{abstract}
We provide the exact time-dependent cosmological solutions in the RandallSundrum (RS) setup with bulk matter, which may be smoothly connected to the static RS metric. In the static limit of the extra dimension, the solutions are reduced to the standard Friedmann equations. In view of our solutions, we also propose an explanation for how the extra dimension is stabilized in spite of a flat modulus potential at the classical level.
\end{abstract}

PACS: 11.25.Mj, 12.10.Dm, 98.80.Cq, 04.50.+h

(Key words: Brane physics, bulk matter, modulus stabilization)

Typeset using REVTEX

*jekim@phyp.snu.ac.kr

${ }^{\dagger}$ kyae@fire.snu.ac.kr 
As a possible solution of the gauge hierarchy problem, Randall and Sundrum (RS) proposed an $S^{1} / Z_{2}$ orbifold model with non-factorizable geometry of space-time [1], which has immediately attracted a great deal of attention. The model employs two branes, Brane 1 (B1) with a positive cosmological constant(or brane tension) $\Lambda_{1} \equiv 6 k_{1} M^{3}$ and Brane 2 (B2) with a negative cosmological constant $\Lambda_{2} \equiv 6 k_{2} M^{3}$, and introduces a negative bulk cosmological constant $\Lambda_{b} \equiv-6 k^{2} M^{3}$. B1 is interpreted as the hidden brane and B2 is identified with the visible brane. Then the metric has an exponential warp factor which could be used to understand the huge gap between the Planck and eletroweak scales. Although the RS setup introduces cosmological constants $k$ in the bulk and $k_{1}$ and $k_{2}$ on the branes, it still describes a static universe because of the fine-tuning between the bulk and brane cosmological constants $k=k_{1}=-k_{2}$, which is a consistency condition in the model. Hence, if the fine-tuning is not exact, the solution has the time dependence and the universe expands exponentially [2] but its form is not suitable for the standard Big Bang universe after the inflation. To circumvent this cosmological problem, some approximation schemes regarding the brane matter in the static limit has been taken into account and(or) some conditions (such as the positive brane tension) for the brane and bulk cosmological constants are required [3]. Thus B1 was thought of as the visible brane [3] even though it is inconsistent with the original motivation for the gauge hierarchy solution [1]. This problem, however, can be resolved with the Gauss-Bonnet interaction [4], bulk matter effects and extra dimension stabilization process [5], etc.

In this paper, we will present exact cosmological solutions in the RS setup with bulk matter. Although there have been many cosmological solutions in the RS setup [2 6], the graceful exit problem from the inflation phase to the standard Big Bang cosmology has not been seriously considered yet. In addition, the role of the extra dimension in the presence of the bulk matter is not well understood. Our exact solutions converge to the RS metric if the space time is made to be static, and leads to the standard Friedmann equations if the fifth dimension is stabilized. In view of our exact solutions, we can find a clue for a stabilization mechanism of the fifth dimension and obtain a small compactified fifth dimension naturally. 
Throughout this paper we consider a $(4+1)$ dimensional universe with coordinate indexed by $(0,1,2,3,5)$. The action describing the bulk matter as well as bulk gravity and brane matter is

$$
S=\int d^{5} x \sqrt{-g}\left(\frac{R}{2}-\Lambda_{b}+\mathcal{L}^{(M)}\right)+\sum_{j=1,2 \text { branes }} \int d^{4} x \sqrt{-g^{(j)}}\left(\mathcal{L}_{j}^{(M)}-\Lambda_{j}\right)
$$

where we set the fundamental scale $M=1 . \mathcal{L}^{(M)}$ and $\mathcal{L}_{j}^{(M)}$ represent matter contributions in the bulk and on the branes. For compatibility with the cosmological principle that our three dimensional space is homogeneous and isotropic, we assume that the metric of the universe has the following form,

$$
d s^{2}=-e^{2 N(\tau, y)} d \tau^{2}+e^{2 A(\tau, y)} \delta_{i j} d x^{i} d x^{j}+e^{2 B(\tau, y)} d y^{2}
$$

where $\tau$ denotes time and $y$ denotes the fifth component. From the metric ansatz, the Einstein tesor $G_{M N}$ is derived through the standard calculation,

$$
\begin{aligned}
& G_{00}^{(1)}=-3 e^{2(N-B)}\left[A^{\prime \prime}+2 A^{\prime 2}-A^{\prime} B^{\prime}\right] \\
& G_{00}^{(2)}=3\left[\dot{A}^{2}+\dot{A} \dot{B}\right] \\
& G_{i i}^{(1)}=e^{2(A-B)}\left[2 A^{\prime \prime}+3 A^{\prime 2}+N^{\prime \prime}+N^{\prime 2}+2 A^{\prime} N^{\prime}-2 A^{\prime} B^{\prime}-B^{\prime} N^{\prime}\right] \\
& G^{(2)}{ }_{i i}=-e^{2(A-N)}\left[2 \ddot{A}+3 \dot{A}^{2}+\ddot{B}+\dot{B}^{2}+2 \dot{A} \dot{B}-2 \dot{A} \dot{N}-\dot{B} \dot{N}\right] \\
& G_{55}^{(1)}=3\left[A^{\prime 2}+A^{\prime} N^{\prime}\right] \\
& G_{55}^{(2)}=-3 e^{2(B-N)}\left[\ddot{A}+2 \dot{A}^{2}-\dot{A} \dot{N}\right] \\
& G_{05}=3\left[A^{\prime} \dot{B}+\dot{A} N^{\prime}-\dot{A}^{\prime}-\dot{A} A^{\prime}\right]
\end{aligned}
$$

where dot and prime denote the derivatives with respect to $\tau$ and $y$, respectively, and the $i$ runs through 1, 2, and 3. Here diagonal Einstein tensors are split into two parts, $G^{(1)}{ }_{A A}$ and $G_{A A}^{(2)}$, depending on the nontrivial $y$ and $\tau$ derivatives, respectively. Thus the original Einstein tensor is, of course, expressed as the sum, $G_{A A} \equiv G_{A A}^{(1)}+G_{A A}^{(2)}$, where the $A$ is $(0, i, 5)$. The source part of the Einstein equation is composed of the cosmological constant and the energy-momentum tensor of matter. In this paper, we will regard the matter as perfect fluid. For the future convenience, we divide also the source tensor into two parts, 


$$
\begin{aligned}
T_{B}^{(1) A}= & -(1-\eta) \cdot \operatorname{diag}\left[\Lambda_{b}, \Lambda_{b}, \Lambda_{b}, \Lambda_{b}, \Lambda_{b}\right] \\
& -\sum_{j=1,2 \text { branes }} \delta\left(y-y_{j}\right) e^{-B} \operatorname{diag}\left[\Lambda_{j}, \Lambda_{j}, \Lambda_{j}, \Lambda_{j}, 0\right] \\
& +\sum_{j=1,2 \text { branes }} \delta\left(y-y_{j}\right) e^{-B} \operatorname{diag}\left[-\hat{\rho}_{j}, \hat{p}_{j}, \hat{p}_{j}, \hat{p}_{j}, 0\right] \\
T^{(2) A}{ }_{B}= & \operatorname{diag}\left[-\left(\rho+\eta \Lambda_{b}\right), P-\eta \Lambda_{b}, P-\eta \Lambda_{b}, P-\eta \Lambda_{b}, P_{5}-\eta \Lambda_{b}\right],
\end{aligned}
$$

where the $\hat{\rho}_{j}$ and $\hat{p}_{j}$ are nontrivial components of the energy-momentum tensor of the matter living only on the $j$-th brane, and $\eta$ is a number representing how $\Lambda_{b}$ is split into $T^{(1)} A_{B}$ and $T^{(2) A}{ }_{B}$. The total source tensor is described as $T^{A}{ }_{B}=T^{(1) A}{ }_{B}+T^{(2) A}{ }_{B}$. Here we set $T_{05}=0$ because it is believed that there is no flow of matter along the fifth direction. The continuity equation of the energy-momentum tensor $T^{A}{ }_{B ; A}=0$ must be satisfied, whose $B=0$ and $B=5$ components are

$$
\begin{aligned}
& \dot{\rho}+3 \dot{A}(\rho+P)+\dot{B}\left(\rho+P_{5}\right)=0 \\
& P_{5}^{\prime}+3 A^{\prime}\left(P_{5}-P\right)+N^{\prime}\left(\rho+P_{5}\right)=0 .
\end{aligned}
$$

The $B=i$ component is identically zero.

Now let us take some ansatze,

$$
\begin{aligned}
& G_{A A}^{(1)}=T_{A A}^{(1)} \quad\left(\text { or } G_{A A}^{(2)}=T_{A A}^{(2)}\right) \\
& A^{\prime}(\tau, y)=N^{\prime}(\tau, y) .
\end{aligned}
$$

The above ansatze have been chosen to fulfill our purpose of restoring the Randall-Sundrum metric in the static limit. The ansatz Eq. (14) and $G_{05}$ read

$$
\begin{aligned}
& 3 e^{-2 B} {\left[A^{\prime \prime}+2 A^{\prime 2}-A^{\prime} B^{\prime}\right] } \\
&=-(1-\eta) \Lambda_{b}-e^{-B}\left[\delta(y)\left(\Lambda_{1}+\rho_{1}\right)+\delta(y-1 / 2)\left(\Lambda_{2}+\rho_{2}\right)\right] \\
& e^{-2 B} {\left[2 A^{\prime \prime}+3 A^{\prime 2}+N^{\prime \prime}+N^{\prime 2}+2 A^{\prime} N^{\prime}-2 A^{\prime} B^{\prime}-B^{\prime} N^{\prime}\right] } \\
&=-(1-\eta) \Lambda_{b}-e^{-B}\left[\delta(y)\left(\Lambda_{1}-p_{1}\right)+\delta(y-1 / 2)\left(\Lambda_{2}-p_{2}\right)\right] \\
& 3 e^{-2 B} {\left[A^{\prime 2}+A^{\prime} N^{\prime}\right]=-(1-\eta) \Lambda_{b} } \\
& A^{\prime} \dot{B}+\dot{A} N^{\prime}-\dot{A}^{\prime}-\dot{A} A^{\prime}=0 .
\end{aligned}
$$


Under the ansatz Eq. (15), Eq. (18) becomes

$$
A^{\prime 2}=-(1-\eta) \frac{\Lambda_{b}}{6} \times e^{2 B} \equiv k^{2} e^{2 B}
$$

Hence, de $\operatorname{Sitter}\left(\Lambda_{b}>0, \eta>1\right)$, anti-de Sitter $\left(\Lambda_{b}<0, \eta<1\right)$, and flat Minkowski space $\left(\Lambda_{b}=0\right)$ are possible in the bulk. The solution consistent with the $S^{1} / Z_{2}$ orbifold symmetry is

$$
A(\tau,|y|) \equiv k F(\tau,|y|)+J(\tau) \quad \text { and } \quad F(\tau,|y|)^{\prime}=-e^{B(\tau,|y|)} \operatorname{sgn}(y)
$$

where the $\operatorname{sgn}(y)$ is defined as $\operatorname{sgn}(y) \equiv|y|^{\prime}=2[\theta(y)-\theta(y-1 / 2)]-1$. Then, because of the ansatz Eq. (15), the exponential factor $N$ of the $g_{00}$ component in our metric tensor is written as

$$
N(\tau,|y|)=k F(\tau,|y|)+K(\tau) \longrightarrow k F(\tau,|y|)
$$

where $K(\tau)$ is removed by the redefinition of time $\tau$ in the second part of the above equation. Therefore, we ignore $K(\tau)$ below.

The above result Eq. (21) leads to some useful relations,

$$
\begin{aligned}
A^{\prime \prime} & =-k e^{B} B^{\prime} \operatorname{sgn}(y)-2[\delta(y)-\delta(y-1 / 2)] k e^{B} \\
& =A^{\prime} B^{\prime}-2[\delta(y)-\delta(y-1 / 2)] k e^{B} \\
\dot{A}^{\prime} & =-k e^{B} \operatorname{sgn}(y) \dot{B}=A^{\prime} \dot{B} .
\end{aligned}
$$

Eq. (24) implies that the $N(\tau,|y|)$ should be stabilized if the $B(\tau,|y|)$ can be stabilized somehow, since $A^{\prime}=N^{\prime}$. With Eqs. (15) and (24), we can show directly that our ansatz is consistent with Eq. (19). Because of Eqs. (15) and (23), Eqs. (16) and (17) just require matching the boundary conditions,

$$
k=\frac{1}{6}\left(\Lambda_{1}+\hat{\rho}_{1}\right)=-\frac{1}{6}\left(\Lambda_{2}+\hat{\rho}_{2}\right) \quad \text { and } \quad \hat{\rho}_{j}=-\hat{p}_{j} .
$$

Hence, considering the fluid continuity equation on the branes, $\dot{\hat{\rho}}_{j}+3 \dot{A}\left(\hat{\rho}_{j}+\hat{p}_{j}\right)=0$, we can arrive at a result $\hat{\rho}_{j}=$ constant and so the $\hat{p}_{j}$ is a constant also, which are expected results 
from our assumption $T_{05}=0$. The non-dynamical properties of the $\hat{\rho}_{j}$ and $\hat{p}_{j}$ obstruct our intention to explain the dynamics of the space-time exactly with brane matter, which is a reason for us to consider bulk matter. [The solution with the brane matter condition $\hat{\rho}_{j}=-\hat{p}_{j}$ is equivalent to that we do not have a solution with the radiation or matter dominated phases by brane matter. Anyway, the radiation or matter dominated universe must results with a vanishing effective cosmological constant, in which case we have not obtained a most general solution.]

Now that we have fulfilled the ansatz Eq. (14) already, the remaining equations, $G^{(2)}{ }_{A A}=$ $T^{(2)}{ }_{A A}$ are

$$
\begin{aligned}
\rho+\eta \Lambda_{b} & =3 e^{-2 N}\left[\dot{A}^{2}+\dot{A} \dot{B}\right] \\
P-\eta \Lambda_{b} & =-e^{-2 N}\left[2 \ddot{A}+3 \dot{A}^{2}+\ddot{B}+\dot{B}^{2}+2 \dot{A} \dot{B}-2 \dot{A} \dot{N}-\dot{B} \dot{N}\right] \\
P_{5}-\eta \Lambda_{b} & =-3 e^{-2 N}\left[\ddot{A}+2 \dot{A}^{2}-\dot{A} \dot{N}\right]
\end{aligned}
$$

which describe the relation between matter and geometry dynamics. They are nothing but the extended Friedmann equations. With Eqs. (15) and (24), we can check that the above equations Eqs. (26), (27) and (28) satisfy both fluid continuity equations, Eqs. (12) and (13) identically, that is, the constraints, Eqs. (19) and (13) are just redundant equations, which are interesting results.] Therefore, the remaining required conditions for the solution are only Eqs. (21) and (22). The relations among the $\rho, P$ and $P_{5}$ may be, of course, governed by particle physics.

Toward a simple solution, let us consider the case that the size of the extra dimension is stabilized, i.e. $\dot{B}=0$, which leads also to $B^{\prime}=0$ generically by redefinition of $y$. Because of Eqs. (15), (22) and (24), then, $\dot{N}$ is also generically set to zero. After all we have

$$
\dot{B}=B^{\prime}=\dot{N}=0
$$

\footnotetext{
1 Compare with the standard cosmology, where two Freedmann equations and one continuity equation lead to one dependent equation.
} 
Then, from Eqs. (21) and (22), the function $F(\tau,|y|)$ is determined to $F(\tau,|y|)=-k e^{B}|y|$ and so

$$
\begin{aligned}
& N(\tau,|y|)=-k e^{B}|y| \equiv-k b_{0}|y| \\
& A(\tau,|y|)=-k b_{0}|y|+J(\tau) \equiv-k b_{0}|y|+\int^{\tau} H(t) d t,
\end{aligned}
$$

where the interval scale $b_{0}$ is a small constant and $H(\tau)$ is a time dependent arbitrary function but may be determined by the equation of state. Thus the metric is read off as

$$
d s^{2}=e^{-2 k b_{0}|y|}\left(-d \tau^{2}+e^{2 \int^{\tau} H(t) d t} d \vec{x}^{2}\right)+b_{0}^{2} d y^{2}
$$

Note that the metric is the same as that of the Randall-Sundrum except for the factor $e^{2 \int^{\tau} H(t) d t}$. Then Eqs. (26), (27) and (28) become

$$
\begin{aligned}
\rho(\tau,|y|)+\eta \Lambda_{b} & =3 e^{2 k b_{0}|y|} H^{2}(\tau) \\
P(\tau,|y|)-\eta \Lambda_{b} & =-e^{2 k b_{0}|y|}\left[2 \dot{H}(\tau)+3 H^{2}(\tau)\right] \\
P_{5}(\tau,|y|)-\eta \Lambda_{b} & =-3 e^{2 k b_{0}|y|}\left[\dot{H}(\tau)+2 H^{2}(\tau)\right] \\
& =-\frac{1}{2}[\rho(\tau,|y|)-3 P(\tau,|y|)]-2 \eta \Lambda_{b} \\
& =\frac{1}{2} T_{\mu}^{(2) \mu},
\end{aligned}
$$

where $\mu$ runs through $0,1,2,3 .[B(\tau,|y|)$ is associated with the vacuum expectation value of a massless four-dimensional scalar field.] The above equations, Eqs. (33), (34) and (35), show that due to the exponential factor matter in the bulk is accumulated mainly near the B2 brane (negative tension brane), which is a similar result to those of the Refs. [7], which are the exact solutions of bulk scalar, gauge boson and fermion fields to their field equations under the fixed RS static background geometry.

Of course, any $H(\tau)$ with $H(\tau) \rightarrow 0$ and $\dot{H}(\tau) \rightarrow 0$ as $\tau \rightarrow \infty$ can lead to an exit from an inflationary phase to a static Randall-Sundrum $(k \neq 0)$ or Minkowski $(k=0)$ universe. In this paper, however, we will not specify a model because we are more interested in the real expanding universe. 
In Eqs.(33), (34) and (35), we should remember that $\rho+\eta \Lambda_{b}, P-\eta \Lambda_{b}$ and $P_{5}-\eta \Lambda_{b}$ are non-trivial components of the 5 dimensional energy-momentum tensor. To derive effective 4 dimensional energy-momentum tensor $\tilde{T}_{B}^{A}$, it is necessary to consider the definition of the original 5 dimensional energy-momentum tensor,

$$
\delta S_{\text {matter }}=\int d^{5} x \sqrt{-g} \delta\left(g_{A}^{B}\right) T_{B}^{(2) A}=\int d^{4} x \int d y b_{0} \sqrt{-g_{4}} \delta\left(\delta_{A}^{B}\right) T_{B}^{(2) A},
$$

where $g_{4} \equiv \operatorname{det}\left[g_{\mu \nu}\right](\mu, \nu=0,1,2,3)$. As the 4 dimensional metric at a 4 dimensional slice in the bulk is $\tilde{g}_{\mu \nu}=e^{2 k b_{0}|y|} g_{\mu \nu}$, which was introduced by Randall and Sundrum to solve the gauge hierarchy problem [1], the effective 4 dimensional energy-momentum tensor is given as

$$
\begin{aligned}
\tilde{T}_{\nu}^{\mu}= & b_{0} \int d y e^{-4 k b_{0}|y|} T_{\nu}^{(2) \mu} \\
= & -b_{0} \int d y e^{-2 k b_{0}|y|} \\
& \quad \cdot \operatorname{diag}\left[3 H^{2}(\tau), 2 \dot{H}(\tau)+3 H^{2}(\tau), 2 \dot{H}(\tau)+3 H^{2}(\tau), 2 \dot{H}(\tau)+3 H^{2}(\tau)\right] \\
\equiv & \operatorname{diag}[-(\tilde{\rho}(\tau)+\eta \tilde{\Lambda}), \tilde{P}(\tau)-\eta \tilde{\Lambda}, \tilde{P}(\tau)-\eta \tilde{\Lambda}, \tilde{P}(\tau)-\eta \tilde{\Lambda}] .
\end{aligned}
$$

According to RS, $b_{0} \int d y e^{-2 k b_{0}|y|}$ is nothing but the induced 4 dimensional Planck scale $M_{P l}^{2}$ [1]. Thus, from Eqs.(33), (34), (37) and (38), we can get the Friedmann equations,

$$
\begin{aligned}
{\left[\frac{\dot{a}(\tau)}{a(\tau)}\right]^{2} } & =\frac{e^{-2 k b_{0}|y|}}{3}\left[\rho(\tau,|y|)+\eta \Lambda_{b}\right]=\frac{1}{3 M_{P l}^{2}}[\tilde{\rho}(\tau)+\eta \tilde{\Lambda}] \quad \text { and } \\
\frac{\ddot{a}(\tau)}{a(\tau)} & =-\frac{e^{-2 k b_{0}|y|}}{6}\left[\rho(\tau,|y|)+3 P(\tau,|y|)-2 \eta \Lambda_{b}\right]=-\frac{1}{6 M_{P l}^{2}}[\tilde{\rho}(\tau)+3 \tilde{P}(\tau)-2 \eta \tilde{\Lambda}],
\end{aligned}
$$

where $a(\tau)$ is a scale factor of our three dimensional space, $a(\tau) \equiv e^{\int^{\tau} H(t) d t}$. Therefore, we could have saved the whole standard cosmological scenario by only requiring stabilization of $B$ in our framework (i.e. $\dot{B}=0$ ). Thus, we do not have to require additional conditions except for $\dot{B}=0$ such as the bulk to have non-zero cosmological constant or the visible brane to have a positive tension, which were essential in other models [3] 6].

As an example, let us consider the vacuum dominated era. The equation of state is $P=-\rho$ and then $P_{5}$ is given by $P_{5}=-2 \rho-\eta \Lambda_{b}$. Then Eqs. (33) and (34) lead to 


$$
H=\text { constant } \equiv H_{0},
$$

which gives the inflationary universe. $H_{0}$ can be considered as a parameter representing the degree of fine-tuning as given in Eq. (33). If $H_{0}$ vanishes, the fine-tuning is successful and the universe is static. On the other hand, a non-zero $H_{0}$ does not satisfy the fine-tuning condition and gives rise to an inflationary universe. Note that our solution is for $\dot{B}=0$ and any modulus potential is not generated at the classical level since $b_{0}$ is arbitrary.

We proceed to discuss the possibility of stabilizing the size of the fifth dimension in our framework. Since we obtained already the solutions for the $\dot{B}=0$ case, we conclude that if $\dot{B}$ goes to zero asymptotically, there exist solutions converging asymptotically to our $\dot{B}=0$ solutions.

We suppose that the stabilization era occurs before or during the conventional inflation era. Thus, we suppose that the system is governed by the same equations of state as those of the inflation era discussed above. Then, from Eqs. (26), (27) and (28), we obtain

$$
\begin{gathered}
2 \ddot{A}+\ddot{B}+\dot{B}^{2}-\dot{A} \dot{B}-2 \dot{A} \dot{N}-\dot{B} \dot{N}=0 \\
\ddot{A}-\dot{A} \dot{N}-2 \dot{A} \dot{B}=0,
\end{gathered}
$$

which are the equations of state in the inflationary era. The above equations and Eqs. (21) and (22) convince us that any potential for the modulus field is not generated also since the $B$ is not fixed yet.

Eq. (43) is easily solved,

$$
\dot{A}=e^{s(|y|)+N} b^{2}=e^{s(|y|)+k F} b^{2}(=k \dot{F}+\dot{J})
$$

where $s(|y|)$ is an integration constant and $b$ is defined as $b(\tau,|y|) \equiv e^{B(\tau,|y|)}$. Note that $A$ is an increasing function of time since $\dot{A}>0$. Removing $\ddot{A}$ from Eqs. (42) and (43), we obtain

$$
\ddot{B}+\dot{B}^{2}+3 \dot{A} \dot{B}-\dot{B} \dot{N}=0
$$

which can be solved to give 


$$
\dot{b}=\frac{1}{e^{t(|y|)-N+3 A}}=\frac{1}{e^{t(|y|)+2 k F+3 J}}
$$

where $t(|y|)$ is an integration constant. Note that $b$ is an increasing function of time but $\dot{b}$ could be zero asymptotically. The extra dimension scale $b$ can be stabilized if the combination $-N+3 A(=2 k F+3 J)$ is an increasing function of time without limit. Especially, if $N \ll A$ and $A$ increases as $\tau \rightarrow \infty$ without a limit at least with a power law, which inflates the three dimensional space, then $\dot{b}$ would decrease exponentially and so b could be stabilized. Below we show that it is possible.

From Eqs. (44) and (46), we obtain

$$
\frac{\dot{A}}{\dot{b}}=e^{s(|y|)+t(|y|)+3 A} b^{2},
$$

which is integrable. The solution is

$$
b(\tau,|y|)^{3}=u^{3}(|y|)-e^{-s(|y|)-t(|y|)-3 A},
$$

where $u(|y|)$ is a $|y|$ dependent arbitrary function. The remaining constraints to satisfy are only Eqs. (21) and (22), i.e. $A^{\prime}=N^{\prime}=-k b(\tau,|y|) \operatorname{sgn}(y)$, from which Eq. (48) can be written as

$$
3 \times \frac{u^{2} u^{\prime}-b^{2} b^{\prime}}{u^{3}(|y|)-b^{3}}+s^{\prime}(|y|)+t^{\prime}(|y|)=-3 A^{\prime}=3 k b \cdot \operatorname{sgn}(y)
$$

We intend to make our solution become the inflationary solution asymptotically that was obtained before. Since $N \rightarrow-k b_{0}|y|, \dot{A} \rightarrow H_{0}$, and $b \rightarrow b_{0}$ as $\tau \rightarrow \infty$, let us take the integration constants in Eqs. (44) and (48) as

$$
\begin{aligned}
& s(|y|)=k b_{0}|y|+\ln H_{0}-2 \ln b_{0} \\
& u(|y|)=b_{0},
\end{aligned}
$$

where $b_{0}$ and $H_{0}$ were defined above. So far the solutions were exact. Note that any value for $H_{0}$ is possible, which does not influence Eq. (49). Although Eq. (49) is difficult to solve exactly, we can argue that $|N|=k|F| \ll\left\{J(\tau)\right.$ and $\left.H_{0} \tau\right\}$ is sufficient to draw a meaningful conclusion. 
$J(\tau)$ and $H_{0}$ can be chosen always such that $|N|=k|F| \ll\left\{J(\tau)\right.$ and $\left.H_{0} \tau\right\}$. It is compatible with the phenomenological requirement of a large $H_{0}$ so that our solutions are derived during the inflationary epoch. (Actually during the inflationary era, $H_{0}^{-1}$ is (a very large value $)^{-1} \approx 10^{-34}$ sec.) In this early inflationary era, $N$ and $b$ are regarded as being much smaller than $H_{0} \tau$, since the scale of the universe was small right after the Big-Bang. Then $-N+3 A$ increases with time and the extra dimension scale $b$ is stabilized rapidly to $b_{0}$. Once $b$ is stabilized, the conditions Eq. (29) become valid and $N$ and $\dot{A}$ are forced to $-k b_{0}|y|$ and $H_{0}$, respectively.

To see our argument explicitly, let us take somewhat large $J(\tau)$ assumption and solve Eqs. (44), (46) and (49) approximately under the condition $k|F| \ll J$. Our aim is to show $\dot{A} \rightarrow H_{0}, \dot{b} \rightarrow 0$, and $b \rightarrow b_{0}$. Here, we set $k F$ and $b$ as $O(1)$ initially, and then $J \gg 1$. Then Eq. (48) gives

$$
b_{0}^{3}-b^{3}(\tau,|y|)=\frac{H_{0}}{b_{0}^{2}} e^{-k b_{0}|y|-3 k F} e^{-3 J} \ll 1 .
$$

With Eqs. (49) and (52) we are led to the following results,

$$
b^{\prime} \ll 1 \quad \text { or } \quad b \approx b(\tau)
$$

and we obtain

$$
F(\tau,|y|) \approx-b(\tau)|y|
$$

Here we must set $t(|y|) \approx 0$ in view of Eq. (46). Then, Eqs. (44) and (46) become

$$
\dot{J} \approx H_{0} \frac{b^{2}}{b_{0}^{2}} \text { and } \quad \dot{b} \approx e^{-3 J} \ll 1,
$$

which shows that during the period of the three space inflation it is quite difficult for $b$ to be dynamical. It is interpreted as the stabilization of the extra dimension in spite of the flat potential for $b$. From Eq. (55) we can derive an expression for $b(\tau)$,

$$
-\frac{1}{6 b_{0}^{2}} \ln \left[\frac{\left(b_{0}-b\right)^{2}}{b_{0}^{2}+b_{0} b+b^{2}}\right]+\frac{1}{b_{0}^{2} \sqrt{3}} \tan ^{-1}\left[\frac{b_{0}+2 b}{b_{0} \sqrt{3}}\right]=\frac{H_{0}}{b_{0}^{2}} \tau,
$$


Or

$$
b(\tau) \approx b_{0}-e^{-3 H_{0} \tau} .
$$

Here we can see that as $\tau \rightarrow \infty, b(\tau)$ grows to $b_{0}$ and $\dot{J}$ tends to $H_{0}$ asymptotically. In other words, to obtain an inflationary universe $b$ should be stabilized to $b_{0}$ exponentially. Note that Eq. (56) becomes an exact result provided the warp factor vanishes, which corresponds the cases of $\Lambda_{b}=0$ or $\eta=1$.

If $b$ is $O(1)$ but small right after the Big Bang, $b_{0}$ should be $O(1)$ but small. As the extra dimension gets stabilized $(\dot{B}=0)$ soon after the beginning of the inflationary era, while the three dimensional space inflates $\left(\right.$ to $\left.e^{H_{0} \tau}\right)$, $b$ remains small $(\approx 1 / M)$ and the universe is reduced effectively to 4-dimension.

In a similar method, we can show that $\dot{b}$ is made asymptotically to zero also in the radiation $(P=\rho / 3, \eta=0)$ and matter dominated era $(P=0, \eta=0)$. However, as the initial condition for $\dot{b}$ is zero (through the above solution in the inflationary epoch), $b$ should have been stabilized already and so Eq. (29) should have been valid since the beginning of the radiation dominated era. Therefore, the Friedmann equations Eqs. (39) and (40) hold good in the radiation and matter dominated eras.

In conclusion, we have provided exact cosmological solutions in the RS setup with bulk matter. In the static limit of all components of the metric, the solutions become the RS metric and in static limit of the extra dimension, they are reduced to the standard Friedmann equations, which implies that bulk matter is accumulated mainly near the negative tension brane (visible brane B2). In this case the modulus potential is not generated effectively at the classical level. With our solution, however, we have shown that the extra dimension could be stabilized (the $\dot{B}=0$ solution) even if the modulus potensial is flat ( $b_{0}$ is arbitrary) and it should be small since the three dimensional space inflates during the inflationary era.

\section{ACKNOWLEDGMENTS}

This work is supported in part by the BK21 program of Ministry of Education. 


\section{REFERENCES}

[1] L. Randall and R. Sundrum, Phys. Rev. Lett. 83, 3370 (1999) hep-ph/9905221.

[2] T. Nihei, Phys. Lett. B465, 81 (1999) hep-ph/9905487; N. Kaloper, Phys. Rev. D60, 123506 (1999) hep-th/9905210; H. B. Kim and H. D. Kim, Phys. Rev. D61, 064003 (2000) hep-ph/9909053; A. Lukas, B. A. Ovrut and D. Waldram, Phys. Rev. D61, 023506 (2000) hep-th/9902071]; H. A. Chamblin and H. S. Reall, Nucl. Phys. B562, 133 (1999) hep-th/9903225; S. Nojiri and S. Odintsov, hep-th/0004097.

[3] P. Binetruy, C. Deffayet and D. Langlois, Nucl. Phys. B565, 269 (2000) hep-th/9905012;

C. Csáki, M Graesser, C. Kolda, and J. Terning, Phys. Lett. B462, 34 (1999) hepph/9906513]; J. M. Cline, C. Grojean and G. Servant, Phys. Rev. Lett. 83, 4245 (1999) [hep-ph/9906523].

[4] J. E. Kim, B. Kyae and H. M. Lee, hep-ph/9912344; ibid. hep-th/0004005.

[5] C. Csáki, M. Graesser, L. Randall, and J. Terning, Phys. Lett. B462, 34 (1999) hepph/9911406; H. B. Kim, Phys. Lett. B478, 285 (2000) hep-th/0001209.

[6] P. Binetruy, C. Deffayet, U. Ellwanger and D. Langlois, Phys. Lett. B477, 285 (2000) [hep-th/9910219]; P. Kanti, K. A. Olive and M. Pospelov, hep-ph/0002229; P. Kanti, I. Kogan, K. A. Olive, and M. Pospelov, Phys. Lett. B468, 31 (1999) hep-ph/9909481; ibid. hep-ph/9912266; L. Mersini, hep-ph/0001017; U. Ellwanger, hep-th/0001126; R. N. Mohapatra, A. Pérez-Lorenzana and C. A. de S. Pires, hep-ph/0003328; J. Lesgourgues, S. Pastor, M. Peloso and L. Sorbo, hep-ph/0004086; A. Lukas, B. A. Ovrut and D. Waldram, Phys. Rev. D60, 086001 (1999) hep-th/9806022; E. E. Flanagan, S.-H. H. Tye and I. Wasserman, hep-ph/9909373; ibid. hep-ph/9910498; H. Stoica, S.-H. H. Tye and I. Wasserman, hep-th/0004126; D. N. Vollick, hep-th/9911181; S. W. Hawking, T. Hetog and R. S. Reall, hep-th/0003052; R. S. Reall, Phys. Rev. D59, 103506 (1999) [hep-th/9809195]. 
[7] W. D. Goldberger and M. B. Wise, Phys. Rev. D60, 107505 (1999) hep-ph/9907218; H. Davoudiasl, J. L. Hewett and T. G. Rizzo, Phys. Lett. B473, 43 (2000) hep-ph/9911262; A. Pomarol, hep-ph/9911294; Y. Grossman and M. Neubert, Phys. Lett. B474, 361 (2000) [hep-ph/9912408]; S. Chang, J. Hisano, H. Nakano, N. Okada and M. Yamaguchi, hep-ph/9912498. 